\title{
Dental considerations in patients with respiratory problems
}

\author{
Ariadna Claramunt Lozano ${ }^{1}, \mathrm{M}^{\mathrm{a}}$ Gracia Sarrión Perez ${ }^{2}$, Carmen Gavaldá Esteve ${ }^{2}$. \\ ${ }^{1}$ Degree in Dental Surgery. Master in Oral Medicine and Surgery. University of Valencia. Valencia, Spain. \\ ${ }^{2}$ Valencia University, Department of Stomatology, University General Hospital, Valencia, Spain.
}

Correspondence:

C/Serpis 66-81

46022 - Valencia (Spain)

E-mail: ariclaramunt@hotmail.com

\begin{abstract}
Many respiratory disorders can compromise routine dental care and require special treatment for the affected patients. Patients often visit the dental clinic with respiratory problems already diagnosed by other specialists. The dental professional therefore must provide correct dental care in the context of such a diagnosis. The present study offers a literature review of those respiratory disorders which can have implications for dental care. Chronic obstructive pulmonary disease (COPD) and asthma require special measures, such as working with the patient in the vertical position, since some of these subjects do not tolerate decubitus. On the other hand, patients with COPD can suffer infectious lung diseases secondary to the aspiration of microorganisms in the presence of deficient periodontal conditions. The treatments received by patients with respiratory diseases can also influence their oral health. In this sense, it has been shown that inhalatory medication used for asthma can cause oral disorders such as xerostomia, oropharyngeal candidiasis and an increased presence of caries (due to the action of $\beta$-agonists), as well as gingivitis. In contrast, oral manifestations of tuberculosis are infrequent. The clinical appearance of the lesions is very similar to that of squamous cell carcinoma; it is therefore important to establish a correct differential diagnosis in such cases. Mention also will be made of patients with obstructive sleep apnea syndrome (OSAS), characterized by critical narrowing and occlusion of the upper airways during sleep. In this context, the dental professional is often directly implicated in the management of such patients by preparing and fitting oral devices designed to advance the mandible. Lastly, mention will be made of dental management in the event of foreign body aspiration, where rapid intervention by the dental professional is critical. The basic approach in such cases is adequate prevention.
\end{abstract}

Key words: Dental treatment, COPD, asthma, tuberculosis, OSAS, foreign body aspiration. 


\section{Introduction}

The respiratory system is basically responsible for $\mathrm{O}_{2}$ and $\mathrm{CO}_{2}$ exchange between the blood and the external environment. This gas exchange takes place passively across partial pressure gradients within the terminal respiratory units (alveolar spaces). Figure 1 schematically represents the structural and functional components of the respiratory system. Maintenance of the mentioned partial pressure gradients is essential for ensuring adequate pulmonary gas exchange (1). Chronic obstructive pulmonary disease (COPD) is an irreversible and slowly progressing disorder characterized by a limitation of airway flow (in some cases partially reversible), resulting from an abnormal pulmonary inflammatory reaction to harmful gases or particles - particularly tobacco smoke. Examples of COPD are chronic bronchitis and lung emphysema. Asthma in turn is a pulmonary disorder characterized by reversible stenosis or stricture of the peripheral bronchi, and is most often seen in children (2). The dental professional must know how to deal with an asthma attack, and must know the drugs which are to be avoided in such patients. Patients with an established diagnosis of tuberculosis (TB) can also be seen in the dental clinic, and the dental professional in any case must be familiarized with the main signs and symptoms of the disease: productive and persistent cough, blood in sputum, nocturnal perspiration, weight loss, fever or anorexia, or a combination of these manifestations. Obstructive sleep apnea syndrome (OSAS) results from intermittent and repeated upper airway occlusion during sleep. The dental professional is often directly implicated in the management of such patients by preparing and fitting oral devices designed to advance the mandible, applying anterior and inferior mandibular traction. On the other hand, dentists often work with small objects or elements, and when the patient is placed in the supine or semi-raised position, such objects might be swallowed or aspirated into the oropharynx. Prevention is clearly the best approach in such cases, though adequate and rapid intervention in the event of accidental aspiration is essential for ensuring patient safety.

\section{Objectives}

The objectives of this study were the following:

- To conduct a literature review of those respiratory disorders which can have implications for dental care.

- To establish protocols for emergency dental management in the context of asthma attacks or the aspiration of foreign bodies.

\section{Material and Methods}

A PubMed - Medline database search was made, and the virtual library of the University of Valencia (Valencia, Spain) and specialized texts in both general Medicine and Odontology were consulted.

The PubMed - Medline search was carried out using the following key words: asthma, lung disease, chronic obstructive pulmonary disease, oral tuberculosis, obstructive sleep apnea, foreign bodies, dental management.

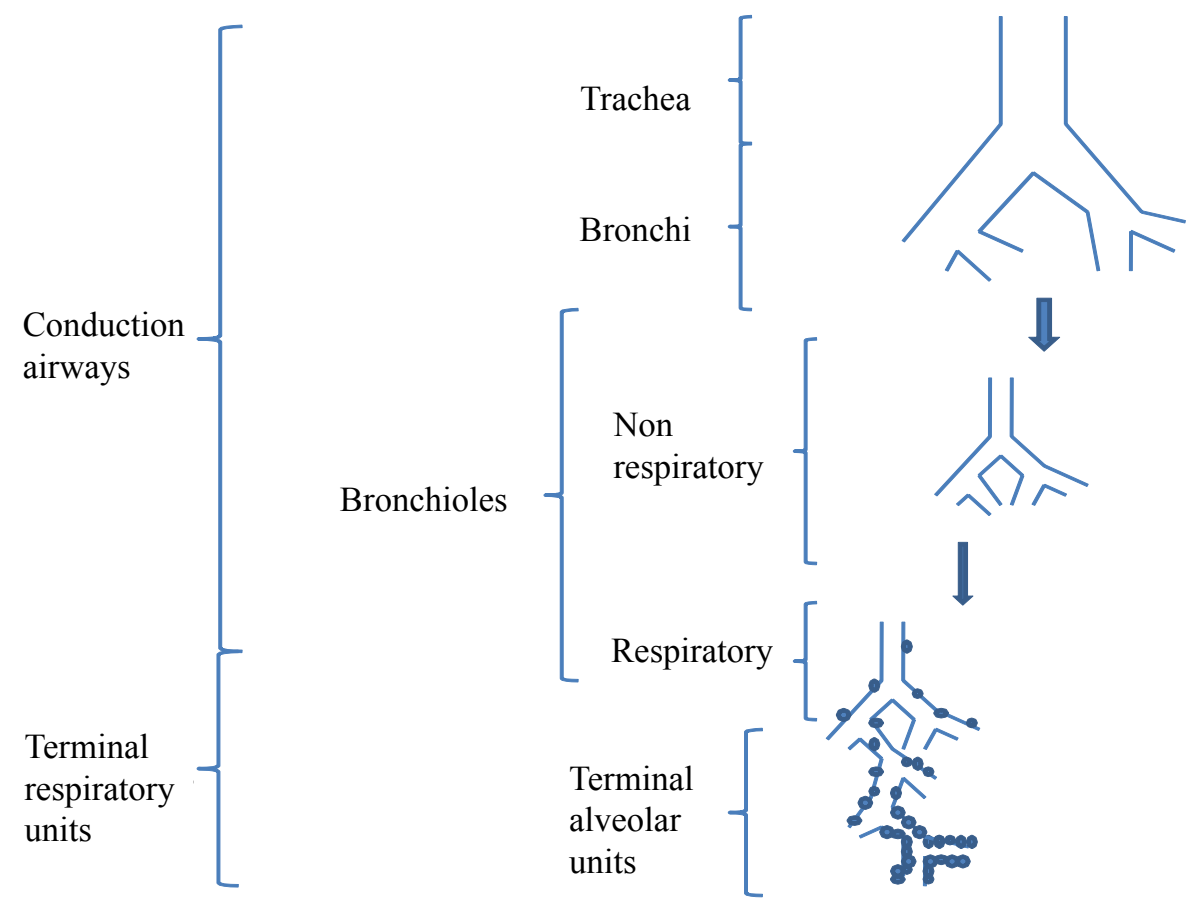

Fig.1. Schematically represents the structural and functional components of the respiratory system. 
The search was limited to articles in English and Spanish, published in dental journals within the last 10 years.

\section{Results}

CHRONIC OBSTRUCTIVE PULMONARY DISEASE (COPD)

Chronic obstructive pulmonary disease (COPD) is a general term used in reference to respiratory disorders characterized by not totally reversible chronic pulmonary airway obstruction. Representative examples of COPD are chronic bronchitis and lung emphysema (3).

COPD is an important public health problem. The prevalence of the disease in the Spanish adult population is $9 \%$, and it constitutes the fourth most common cause of death in Spain and in the rest of the world (3).

Patients with COPD may experience worsening of respiratory function during dental treatment; a number of precautions are therefore recommended. Specifically, it is advisable to treat the patient in the vertical position. The way in which rubber dams are used should also be modified in some cases, since the patients may complain that they produce a suffocating sensation. Specialized clinics are able to offer oxygen equipment and personnel trained in its use. Hypnotics, narcotics, antihistamines and anticholinergic agents are to be avoided. If the patient is receiving corticosteroids, supplements may be needed. In the case of individuals receiving theophylline, macrolide antibiotics (erythromycin, clarithromycin) are to be avoided. Ambulatory general anesthesia is totally contraindicated.

On the other hand, patients with COPD, particularly those admitted to hospital, can suffer infectious lung diseases secondary to the aspiration of microorganisms in the presence of deficient periodontal conditions. The teeth and periodontium can serve as a reservoir for respiratory infections. In this sense, loss of alveolar bone has been associated to an increased risk of COPD $(4,5)$. Smoking is an important risk factor for both periodontitis and COPD.

Regarding the impact of respiratory infection upon periodontal health, no studies to date have established a direct or indirect association between the two conditions (4).

ASTHMA

Asthma is a respiratory disease characterized by reversible, diffuse stenosis or stricture of the peripheral bronchi, increased responsiveness or sensitivity to different stimuli, and frequently also signs or laboratory test evidence of an allergic alteration. Asthma is a common condition, typically affecting children and with a prevalence of 5-6\% (2). Over half of all affected individuals are between 5-15 years of age. A distinction is to be made between allergic and non-allergic asthma.

Allergic (or extrinsic) asthma is characterized by a family history of asthma, together with an increase in serum IgE titers. These antibodies participate in type I hypersensitivity or immediate sensitivity reactions, and are produced in response to exposure to antigens that access the body through the oral or parenteral route, or in aerosol form.

Non-allergic, idiosyncratic or intrinsic asthma in turn constitutes a respiratory disorder manifesting in a heterogeneous group of patients with reversible and recurrent bronchospasm in response to different stimuli such as physical exercise, the inhalation of cold air, emotions, exposure to smoke, hypoxemia, stress, gastroesophageal reflux, etc. (2). Patients with this type of asthma can be sensitive to aspirin and nonsteroidal antiinflammatory drugs (NSAIDs).

The drugs used to treat asthma have been related to certain oral disorders such as xerostomia (dry mouth), oropharyngeal candidiasis and an increased prevalence of caries (due to the use of inhalatory $\beta$-agonists)(6-8). The use of oral rinses after medication has been found to be of great help in preventing oral mucosal alterations. Asthmatic patients can also suffer gingivitis, since they are often oral breathers, and this condition together with a number of immunological factors can contribute to increase gingival inflammation (8).

Children with chronic medical problems requiring longterm medication are at an increased risk of developing caries as a side effect of the treatment received. A pos-

\begin{tabular}{l} 
DRUGS TO BE AVOIDED IN ASTHMATIC PATIENTS \\
\hline Drugs containing aspirin (10-28\% of all asthmatics may not tolerate the latter)(2). \\
\hline Nonsteroidal antiinflammatory drugs (patients with intrinsic asthma). \\
\hline $\begin{array}{l}\text { Macrolide antibiotics in patients treated with theophylline. The serum methylxanthines levels (theophylli- } \\
\text { ne) may be increased. }\end{array}$ \\
\hline Opiates: these can cause respiratory depression and histamine release. \\
\hline $\begin{array}{l}\text { Local anesthetics: use solutions without adrenalin or levonordefrin, due to the sulfite preservative con- } \\
\text { tents. }\end{array}$ \\
\hline $\begin{array}{l}\text { If the patient is receiving prolonged systemic corticosteroid treatment, supplements may be needed (prior } \\
\text { to dental procedures that might cause stress). }\end{array}$ \\
\hline
\end{tabular}

Table 1. Drugs to be avoided in asthmatic patients. 
sible mechanism implicated in the development of caries could be the intervention of $\beta$-agonists, exerting an effect also upon the salivary glands. The impairment of salivary secretion is in direct relation to the drug dose, and the composition of saliva is also affected. Different studies have reported an increase in dental caries in children treated with inhalatory salbutamol, followed by salbutamol in tablets, as a result of prolonged exposure to such $\beta 2$-agonists, which reduce salivary secretion $(7,8)$. Caries also result from an increased presence of lactobacilli and Streptococcus mutans. Periodic dental examinations and the application of fluor are advised in these individuals. Elective treatment should be carried out in asymptomatic or controlled asthmatic patients (8). Table 1 shows a number of drugs used in dental care and which have specific implications when dealing with asthmatic patients (6).

Management of asthma attacks:

Breathing effort becomes audible in the presence of mild or moderate obstruction. The typical symptoms of asthma are breathing difficulty (e.g., wheezing, dyspnea) and cough $(2,8)$. On the other hand, the simple establishment of an optimum patient-professional relationship contributes to minimize the stress and nervousness associated to dental treatment. The critical moments of dental treatment in which an asthma attack can be triggered are immediately after local anesthetic injection and those maneuvers that cause stress - such as extractions, surgery, or dental pulp removal in endodontic procedures (8). Table 2 presents the recommended guidelines during an asthma attack.

PULMONARY TUBERCULOSIS

Tuberculosis (TB) is one of the main causes of death throughout the world. Approximately one-third of the world population is infected with Mycobacterium tuberculosis (9-11). Tuberculosis can affect any body organ, though the lungs are the most common location. At first exposure to the bacterium (primary infection with the Koch bacillus), the latter induces a characteristic granulomatous reaction (tuberculous follicle or granuloma). Oral tuberculosis:

Oral mucosal lesions of TB are more common in young patients (10). Only $0.05-5 \%$ of all cases of tuberculosis involve oral manifestations. These lesions may be primary or (more often) secondary to pulmonary tuberculosis $(12,13)$, reflecting reactivation of the infection at a given moment, since the bacteria remain latent even after clinical healing of the initial infection (12). The oral lesions manifest as an irregular ulceration with polygonal margins, possible peripheral induration and a dirtyappearing base. The clinical appearance of the lesions is very similar to that of squamous cell carcinoma (11); it is therefore important to establish a correct differential diagnosis in such cases, based on a biopsy and bacterial culture. Oral TB has been reported in mandibular bone, the tongue and, less frequently, affecting the lips, palatine tonsils and posterior pharyngeal wall (13) and parotid glands $(11,12)$. There have also been reports of cases in other soft tissue locations such as the cheek.

When TB is suspected, it is advisable to postpone all non-emergency dental treatment until the patient has been cured or is no longer infectious $(9,11)$.

If emergency dental treatment proves necessary in patients with suspected TB or active disease, the adoption of respiratory protection measures helps reduce the risk of exposure. The dental professional must avoid inhaling the infectious droplets by wearing a protective face mask.

\section{OBSTRUCTIVE SLEEP APNEA SYNDROME (OSAS)}

Obstructive sleep apnea syndrome (OSAS) results from intermittent and repeated upper airway occlusion during sleep. Such occlusion results from inspiratory collapse of the pharyngeal walls, with complete (apnea) or partial interruption of the airflow (hypopnea). Such apneic or hypopneic episodes are of variable duration and have a different effect upon cardiorespiratory homeostasis (14).

Recent studies carried out in Spain have reported prevalences of OSAS of between $4-6 \%$ in adult males and about $2 \%$ in women (15).

Regarding the physiopathology of OSAS, critical upper airway narrowing during sleep results in occlusion. This phenomenon in turn is intensified in obese people or individuals with other respiratory disorders such as asthma or COPD. Following obstruction, the sleeping patient may attempt to increase the air inflow rate in or-

MANAGEMENT OF ASTHMA ATTACKS
\begin{tabular}{|l|}
\hline 1. Suspend the dental procedure and raise the patient to a comfortable position. \\
\hline 2. Establish and keep the airways free, and administer an inhalatory $\beta 2$ agonist. \\
\hline $\begin{array}{l}\text { 3. Administer oxygen with a mask. If no improvement is observed or the symptoms worsen, administer } \\
\text { subcutaneous epinephrine (1:1000 in solution, } 0.01 \mathrm{mg} / \mathrm{kg} \text { body weight, with a maximum dose of } 0.3 \\
\mathrm{mg} \text { ). }\end{array}$ \\
\hline 4. Notify the emergency medical service. \\
\hline 5. Maintain adequate oxygen levels until the patient breathes regularly and/or medical help arrives $(8)$. \\
\hline
\end{tabular}




\section{APPROACH TO FOREIGN BODY ASPIRATION}

1. Raise the patient and instruct him or her to cough forcefully.

2. If breathing is affected (asphyxia, inspiratory stridor and the need to breathe with accessory muscle support) and vigorous coughing proves ineffective, perform the Heimlich maneuver.

3. If this likewise proves ineffective, notify the emergency medical service immediately. While waiting for patient transfer, apply vital support measures, including airway permeation by means of a cricothyroidotomy, where necessary.

4. If the airway is not affected, the swallowed object should be recovered to calm the patient.

Table 3. Approach to foreign body aspiration.

der to maintain the required oxygen supply, resulting in noisy, intense and increasing snoring that causes partial or complete awakening. This in turn leads to tiredness and drowsiness in the daytime (14), together with a series of psychomotor effects that depend on the severity of hypoxemia and the duration of drowsiness.

At dental exploration, palpation of the muscles of the head and neck is indicated in order to identify possible masses or tumors that might be the cause of airway obstruction. Other possible contributing conditions are deviation of the nasal septum (16), class II malocclusion, large gonial angles, the size of the tongue and its base, and the oropharynx (size of the uvula, size and characteristics of the soft palate tissues).

The dental professional is often directly implicated in the management of patients with OSAS by preparing and fitting oral devices designed to advance the mandible, applying anterior and inferior mandibular traction (17). The basic purpose of these devices is to prevent the base of the tongue from coming too close to the posterior oropharyngeal region, where obstruction can result.

- Tongue retainer: this device positions the tongue in a cup or bubble located between the anterior teeth. The tongue is not maintained in this position for long during sleep; as a result, this type of device is indicated in patients with brief periods of apnea.

- Mandibular advancing device: this element stabilizes the mandible both vertically and horizontally. It also keeps the tongue away from the pharyngeal wall, since mandibular protrusion induces relative advancement of the tongue (due to the insertion of the genioglossal muscle)(14,18).

Given the relevance of respiratory disorders such as OSAS, dental professionals should be able to promptly identify and treat the condition, thereby contributing to the integral management of these patients.

FOREIGN BODY ASPIRATION

Many dental materials and elements are of small size, and when exposed to saliva it may be difficult to manipulate them correctly. When the patient is placed in the supine or semi-raised position, such objects might be swallowed or aspirated into the oropharynx. De- pending on the size, shape and flexibility of the object, swallowing may pose only minimum risk or potentially can prove fatal. Prevention is clearly the best approach in such cases, though adequate and rapid intervention in the event of accidental aspiration is essential for ensuring patient safety.

When a foreign body is aspirated into the oropharynx, the patient should sit up and be instructed to cough forcefully. The immediate priority is to ensure that the airways remain free. If breathing is affected, clearly recognizable symptoms quickly develop, such as asphyxia, inspiratory stridor and the need to breathe with accessory muscle support. If vigorous coughing is not effective, the Heimlich maneuver should be used: with the patient in the standing position, we grasp him or her from behind with both arms. In this position we apply pressure with one closed fist and the other hand covering the fist. The fist is positioned with the thumb over the abdomen, and we press firmly towards the center of the stomach, immediately below the ribcage. If this maneuver likewise proves ineffective, the patient must be moved to the nearest emergency medical center as quickly as possible. While waiting for patient transfer, the dental professional should apply vital support measures, including airway permeation by means of a cricothyroidotomy, where necessary.

If the airway is not affected, the swallowed object should be recovered to thus calm the patient. If retrieval of the object is not possible, the situation should be explained to the patient, and due chest and abdominal X-rays and clinical evaluation in the hospital will help identify the location of the object (19)(Table 3).

\section{Conclusion}

The main disease states that can give rise to respiratory problems during dental treatment procedures are COPD, asthma, tuberculosis, OSAS and foreign body aspiration. The dental professional must know these diseases in order to be able to offer effective and safe treatment, and must be able to recognize the oral and/or dental manifestations that might arise.

Asthma attacks and foreign body aspiration are the two 
emergency situations that can be seen when treating patients of this kind. The intervention protocols applicable to such situations are summarized in Tables 2 and 3.

\section{References}

1. Weibel ER. What makes a good lung? Swiss Med Wkly. 2009; 139:375-86.

2. Sollecito TP, Tino G. Asthma. Oral Surg Oral Med Oral Pathol Oral Radiol Endod. 2001;92:485-90.

3. Peces-Barba G, Barberà JA, Agustí A, Casanova C, Casas A, Izquierdo JL, et al. Diagnosis and management of chronic obstructive pulmonary disease: joint guidelines of the Spanish Society of Pulmonology and Thoracic Surgery (SEPAR) and the Latin American Thoracic Society (ALAT). Arch Bronconeumol. 2008;44:271-81.

4. Kuo LC, Polson AM, Kang T. Associations between periodontal diseases and systemic diseases: A review of the inter-relationships and interactions with diabetes, respiratory diseases, cardiovascular diseases and osteoporosis. Public Health. 2008;12:417-33.

5. Leuckfeld I, Obregon-Whittle MV, Lund MB, Geiran O, Bjørtu$\mathrm{ft} \mathrm{O}$, Olsen I. Severe chronic obstructive pulmonary disease: Association with marginal bone loss in periodontitis. Respir Med. 2008;102, 488-94.

6. Laurikainen K, Kuusisto P. Comparison of the oral health status and salivary flow rate of asthmatic patients with those of nonasthmatic adults - results of a pilot study. Allergy. 1998;53:316-9.

7. Shashikiran ND, Reddy VV, Raju PK. Effect of antiasthmatic medication on dental disease: dental caries and periodontal disease. J Indian Soc Pedod Prev Dent. 2007;25:65-8.

8. Steinbacher DM, Glick M. The dental patient with asthma. An update and oral health considerations. J Am Dent Assoc. 2001;132:1229-39.

9. Cleveland JL, Robison VA, Panlilio AL. Tuberculosis epidemiology, diagnosis and infection control recommendations for dental settings: an update on the Centers for Disease Control and Prevention guidelines. J Am Dent Assoc. 2009;140:1092-9.

10. Tovaru S, Costache M, Sardella A. Primary oral tuberculosis: a case series from Bucharest, Romania. Oral Surg Oral Med Oral Pathol Oral Radiol Endod. 2008;105:e41-5.

11. Ajay GN, Laxmikanth C, Prashanth SK. Tuberculous ulcer of tongue with oral complications of oral antituberculosis therapy. Indian J Dent Res. 2006;17:87-90.

12. Ozcan C, Apa DD, Aslan G, Gülhan S, Görür K. Mycobacterium tuberculosis infection within parotid gland Warthin tumor. J Craniofac Surg. 2008;19:1561-5.

13. Gupta PP, Fotedar S, Agarwal D, Sansanwal P. Primary tuberculous glossitis in an immunocompetent patient. Hong Kong Med J. 2007;13:330-1.

14. Rodríguez-Lozano FJ, Sáez-Yuguero Mdel R, Linares Tovar E, Bermejo Fenoll A. Sleep apnea and mandibular advancement device. Revision of the literature. Med Oral Patol Oral Cir Bucal. 2008;13:549-54.

15. Stradling JR, Davies RJ. Sleep. 1: Obstructive sleep apnoea/hypopnoea syndrome: definitions, epidemiology, and natural history. Thorax. 2004;59:73-8.

16. Magliocca KR, Helman JI. Obstructive sleep apnea: diagnosis, medical management and dental implications. J Am Dent Assoc. 2005;136:1121-9.

17. Lawton HM, Battagel JM, Kotecha B. A comparison of the Twin Block and Herbst mandibular advancement splints in the treatment of patients with obstructive sleep apnoea: a prospective study. Eur J Orthod. 2005;27:82-90.

18. Tsuiki S, Lowe AA, Almeida FR, Kawahata N, Fleetham JA. Effects of mandibular advancement on airway curvature and obstructive sleep apnoea severity. Eur Respir J. 2004;23:263-8.

19. Milton TM, Hearing SD, Ireland AJ. Ingested foreign bodies associated with orthodontic treatment: report of three cases and review of ingestion/aspiration incident management. Br Dent J. 2001;190:592-6. 